\title{
Incorporating Quotation and Evaluation into Church's Type Theory: Syntax and Semantics ${ }^{\star}$
}

\author{
William M. Farmer \\ Computing and Software, McMaster University, Canada \\ wmf armer@mcmaster.ca
}

27 July 2016

\begin{abstract}
CTT $_{\mathrm{qe}}$ is a version of Church's type theory that includes quotation and evaluation operators that are similar to quote and eval in the Lisp programming language. With quotation and evaluation it is possible to reason in $\mathrm{CTT}_{\mathrm{qe}}$ about the interplay of the syntax and semantics of expressions and, as a result, to formalize syntax-based mathematical algorithms. We present the syntax and semantics of $\mathrm{CTT}_{\mathrm{qe}}$ and give several examples that illustrate the usefulness of having quotation and evaluation in $\mathrm{CTT}_{\mathrm{qe}}$. We do not give a proof system for $\mathrm{CTT}_{\mathrm{qe}}$, but we do sketch what a proof system could look like.
\end{abstract}

\section{Introduction}

The Lisp programming language is famous for its use of quotation and evaluation. From code the Lisp quotation operator called quote produces meta-level data (i.e., S-expressions) that represents the code, and from this data the Lisp evaluation operator called eval produces the code that the data represents. In Lisp, metaprogramming (i.e., programming at the meta-level) is performed by manipulating S-expressions and is reflected (i.e., integrated) into object-level programming by the use of quote and eval.

Metaprogramming with reflection is a very powerful programming tool. Besides Lisp, several other programming languages employ quotation and evaluation mechanisms to enable metaprogramming with reflection. Examples include Agda [16 17, Archon [22, Elixir [18, F\# 25], MetaML [23, MetaOCaml 20], reFLect [12], and Template Haskell [21].

Analogous to metaprogramming in a programming language, metareasoning is performed in a logic by manipulating meta-level values (e.g., syntax trees) that represent expressions in the logic and is reflected into object-level reasoning

\footnotetext{
* Published in: M. Kohlhase et al., eds, Intelligent Computer Mathematics (CICM 2016), Lecture Notes in Computer Science, Vol. 9791, pp. 8398, Springer, 2016. The final publication is available at Springer via http://dx.doi.org/10.1007/978-3-319-42547-4_7. This research was supported by NSERC.
} 
using quotation and evaluation 1 mechanisms [6]. In proof assistants like Coq and Agda, metareasoning with reflection is implemented in the logic by defining an infrastructure consisting of (1) an inductive type of syntactic values that represent certain object-level expressions, (2) an informal quotation operator that maps these object-level expressions to syntactic values, and (3) a formal evaluation operator that maps syntactic values to the values of the object-level expressions that they represent [4]10/26]. Metareasoning with reflection is used for formalizing metalogical techniques and incorporating symbolic computation into proof assistants [48/1013/26].

The metareasoning and reflection infrastructures that have been employed in today's proof assistants are local in the sense that the syntactic values of the inductive type represent only a subset of the expressions of the logic, the quotation operator can only be applied to these expressions, and the evaluation operator can only be applied to the syntactic values of the inductive type. Can metareasoning with reflection be implemented in a traditional logic like firstorder logic or simple type theory using a global infrastructure with quotation and evaluation operators like Lisp's quote and eval? This is largely an open question. As far as we know, there is no readily implementable version of a traditional logic that admits global quotation and evaluation. We have proposed a version of NBG set theory named Chiron [7] and a version of Alonzo Church's type theory $[5]$ named $\mathcal{Q}_{0}^{\text {uqe }} 9$ that include global quotation and evaluation operators, but these logics have a high level of complexity and are not easy to implement.

Many challenging problems face the logic engineer who seeks to incorporate global quotation and evaluation into a traditional logic. The three problems that most concern us are the following. We will write the quotation and evaluation

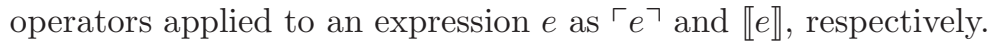

1. Evaluation Problem. An evaluation operator is applicable to syntactic values that represent formulas and thus is effectively a truth predicate. Hence, by the proof of Alfred Tarski's theorem on the undefinability of truth 24, if the evaluation operator is total in the context of a sufficiently strong theory like first-order Peano arithmetic, then it is possible to express the liar paradox using the quotation and evaluation operators. Therefore, the evaluation operator must be partial and the law of disquotation cannot hold universally (i.e., for some expressions $e, \llbracket\ulcorner e\urcorner \rrbracket \neq e$ ). As a result, reasoning with evaluation is cumbersome and leads to undefined expressions.

2. Variable Problem. The variable $x$ is not free in the expression $\ulcorner x+3\urcorner$ (or in any quotation). However, $x$ is free in $\llbracket\ulcorner x+3\urcorner \rrbracket$ because $\llbracket\ulcorner x+3\urcorner \rrbracket=x+3$. If the value of a constant $c$ is $\ulcorner x+3\urcorner$, then $x$ is free in $\llbracket c \rrbracket$ because $\llbracket c \rrbracket=$ $\llbracket\ulcorner x+3\urcorner \rrbracket=x+3$. Hence, in the presence of an evaluation operator, whether or not a variable is free in an expression may depend on the values of the expression's components. As a consequence, the substitution of an expression

\footnotetext{
${ }^{1}$ Evaluation in this context is also called unquoting, interpretation, dereferencing, and dereification.

${ }^{2}$ Church's type theory is a version of simple type theory with lambda notation.
} 
for the free occurrences of a variable in another expression depends on the semantics (as well as the syntax) of the expressions involved and must be integrated with the proof system of the logic. That is, a logic with quotation and evaluation requires a semantics-dependent form of substitution in which side conditions, like whether a variable is free in an expression, are proved within the proof system. This is a major departure from traditional logic.

3. Double Substitution Problem. By the semantics of evaluation, the value of $\llbracket e \rrbracket$ is the value of the expression whose syntax tree is represented by the value of $e$. Hence the semantics of evaluation involves a double valuation (see condition 6 of the definition of a model in section 3.3. $)$. If the value of a variable $x$ is $\ulcorner x\urcorner$, then $\llbracket x \rrbracket=\llbracket\ulcorner x\urcorner \rrbracket=x=\ulcorner x\urcorner$. Hence the substitution of $\ulcorner x\urcorner$ for $x$ in $\llbracket x \rrbracket$ requires one substitution inside the argument of the evaluation operator and another substitution after the evaluation operator is eliminated. This double substitution is another major departure from traditional logic.

$\mathrm{CTT}_{\mathrm{qe}}$ is a version of Church's type theory [5] with quotation and evaluation that overcomes these three problems. It is much simpler than $\mathcal{Q}_{0}^{\text {uqe }}$ since (1) the quotation operator can only be applied to expressions that do not contain the evaluation operator and (2) substitution is not a logical constant (applied to syntactic values). Like $\mathcal{Q}_{0}^{\text {uqe }}, \mathrm{CTT}_{\mathrm{qe}}$ is based on $\mathcal{Q}_{0}$ [2], Peter Andrews' version of Church's type theory. In this paper, we present the syntax and semantics of $\mathrm{CTT}_{\mathrm{qe}}$ and give several examples that illustrate the usefulness of having quotation and evaluation in $\mathrm{CTT}_{\mathrm{qe}}$. We do not give a proof system for $\mathrm{CTT}_{\mathrm{qe}}$, but we do sketch what a proof system could look like.

\section{Syntax}

The syntax of $\mathrm{CTT}_{\mathrm{qe}}$ is very similar to the syntax of $\mathcal{Q}_{0}$ [2, pp. 210-211]. $\mathrm{CTT}_{\mathrm{qe}}$ has the syntax of Church's type theory plus an inductive type of syntactic values, a quotation operator, and a typed evaluation operator. Like $\mathcal{Q}_{0}$, the propositional connectives and quantifiers are defined using function application, function abstraction, and equality. For the sake of simplicity, $\mathrm{CTT}_{\mathrm{qe}}$ does not contain, as in $\mathcal{Q}_{0}$, a definite description operator or, as in the logic of HOL [11, an indefinite description (choice) operator or type variables.

\section{$2.1 \quad$ Types}

A type of $\mathrm{CTT}_{\mathrm{qe}}$ is a string of symbols defined inductively by the following formation rules:

1. Type of individuals: $\iota$ is a type.

2. Type of truth values: $o$ is a type.

3. Type of constructions: $\epsilon$ is a type.

4. Function type: If $\alpha$ and $\beta$ are types, then $(\alpha \rightarrow \beta)$ is a type 3

\footnotetext{
${ }^{3}$ In Andrews' $\mathcal{Q}_{0}[2]$ and Church's original system [5], the function type $(\alpha \rightarrow \beta)$ is written as $(\beta \alpha)$.
} 
We assume that function application formation associates to the left so that an expression of the form $\left(\left(\mathbf{G}_{\alpha \rightarrow \beta \rightarrow \gamma} \mathbf{A}_{\alpha}\right) \mathbf{B}_{\beta}\right)$ may be written as $\mathbf{G}_{\alpha \rightarrow \beta \rightarrow \gamma} \mathbf{A}_{\alpha} \mathbf{B}_{\beta}$.

\subsection{Constructions}

A construction of $\mathrm{CTT}_{\mathrm{qe}}$ is an expression of type $\epsilon$ defined inductively as follows:

1. $\left\ulcorner\mathbf{x}_{\alpha}\right\urcorner$ is a construction.

2. $\left\ulcorner\mathbf{c}_{\alpha}\right\urcorner$ is a construction.

3. If $\mathbf{A}_{\epsilon}$ and $\mathbf{B}_{\epsilon}$ are constructions, then app $\operatorname{apt\epsilon \rightarrow \epsilon }_{\epsilon} \mathbf{A}_{\epsilon} \mathbf{B}_{\epsilon}$, abs $_{\epsilon \rightarrow \epsilon \rightarrow \epsilon} \mathbf{A}_{\epsilon} \mathbf{B}_{\epsilon}$, and quo $_{\epsilon \rightarrow \epsilon} \mathbf{A}_{\epsilon}$ are constructions.

The set of constructions is thus an inductive type whose base elements are quotations of variables and constants and whose constructors are app $\operatorname{apt\epsilon \rightarrow \epsilon }_{\epsilon}$, abs $_{\epsilon \rightarrow \epsilon \rightarrow \epsilon}$, and quo $_{\epsilon \rightarrow \epsilon}$. We will call these three constants syntax constructors.

Let $\mathcal{E}$ be the function mapping eval-free expressions to constructions that is defined inductively as follows:

1. $\mathcal{E}\left(\mathbf{x}_{\alpha}\right)=\left\ulcorner\mathbf{x}_{\alpha}\right\urcorner$.

2. $\mathcal{E}\left(\mathbf{c}_{\alpha}\right)=\left\ulcorner\mathbf{c}_{\alpha}\right\urcorner$.

3. $\mathcal{E}\left(\mathbf{F}_{\alpha \rightarrow \beta} \mathbf{A}_{\alpha}\right)=\operatorname{app}_{\epsilon \rightarrow \epsilon \rightarrow \epsilon} \mathcal{E}\left(\mathbf{F}_{\alpha \rightarrow \beta}\right) \mathcal{E}\left(\mathbf{A}_{\alpha}\right)$.

4. $\mathcal{E}\left(\lambda \mathbf{x}_{\alpha} . \mathbf{B}_{\beta}\right)=\mathrm{abs}_{\epsilon \rightarrow \epsilon \rightarrow \epsilon} \mathcal{E}\left(\mathbf{x}_{\alpha}\right) \mathcal{E}\left(\mathbf{B}_{\beta}\right)$.

5. $\mathcal{E}\left(\left\ulcorner\mathbf{A}_{\alpha}\right\urcorner\right)=$ quo $_{\epsilon \rightarrow \epsilon} \mathcal{E}\left(\mathbf{A}_{\alpha}\right)$.

$\mathcal{E}$ is clearly injective. When $\mathbf{A}_{\alpha}$ is eval-free, $\mathcal{E}\left(\mathbf{A}_{\alpha}\right)$ is a construction that represents the syntax tree of $\mathbf{A}_{\alpha}$. That is, $\mathcal{E}\left(\mathbf{A}_{\alpha}\right)$ is a syntactic value that represents how $\mathbf{A}_{\alpha}$ is syntactically constructed. For every eval-free expression, there is a construction that represents its syntax tree, but not every construction represents the syntax tree of an eval-free expression. For example, app $\cos _{\epsilon \rightarrow \epsilon}\left\ulcorner\mathbf{x}_{\alpha}\right\urcorner\left\ulcorner\mathbf{x}_{\alpha}\right\urcorner$ represents the syntax tree of $\left(\mathbf{x}_{\alpha} \mathbf{x}_{\alpha}\right)$ which is not an expression of $\mathrm{CTT}_{\mathrm{qe}}$ since the types are mismatched. A construction is proper if it is in the range of $\mathcal{E}$, i.e., it represents the syntax tree of an eval-free expression.

The five kinds of eval-free expressions and the syntactic values that represent their syntax trees are given in Table 2 .

\begin{tabular}{|lll|}
\hline Kind & Syntax & Syntactic Values \\
Variable & $\mathbf{x}_{\alpha}$ & $\left\ulcorner\mathbf{x}_{\alpha}\right\urcorner$ \\
Constant & $\mathbf{c}_{\alpha}$ & $\left\ulcorner\mathbf{c}_{\alpha}\right\urcorner$ \\
Function application & $\mathbf{F}_{\alpha \rightarrow \beta} \mathbf{A}_{\alpha}$ & $\operatorname{app}_{\epsilon \rightarrow \epsilon \rightarrow \epsilon} \mathcal{E}\left(\mathbf{F}_{\alpha \rightarrow \beta}\right) \mathcal{E}\left(\mathbf{A}_{\alpha}\right)$ \\
Function abstraction & $\lambda \mathbf{x}_{\alpha} \cdot \mathbf{B}_{\beta}$ & $\operatorname{abs}_{\epsilon \rightarrow \epsilon \rightarrow \epsilon} \mathcal{E}\left(\mathbf{x}_{\alpha}\right) \mathcal{E}\left(\mathbf{B}_{\beta}\right)$ \\
Quotation & $\left\ulcorner\mathbf{A}_{\alpha}\right\urcorner$ & quo $_{\epsilon \rightarrow \epsilon} \mathcal{E}\left(\mathbf{A}_{\alpha}\right)$ \\
\hline
\end{tabular}

Table 2. Five Kinds of Eval-Free Expressions 


\subsection{Definitions and Abbreviations}

As Andrews does in [2, p. 212], we introduce in Table 3 several defined logical constants and abbreviations. The former includes constants for true and false and the propositional connectives. The latter includes notation for equality, the propositional connectives, universal and existential quantification, and a simplified notation for evaluations.

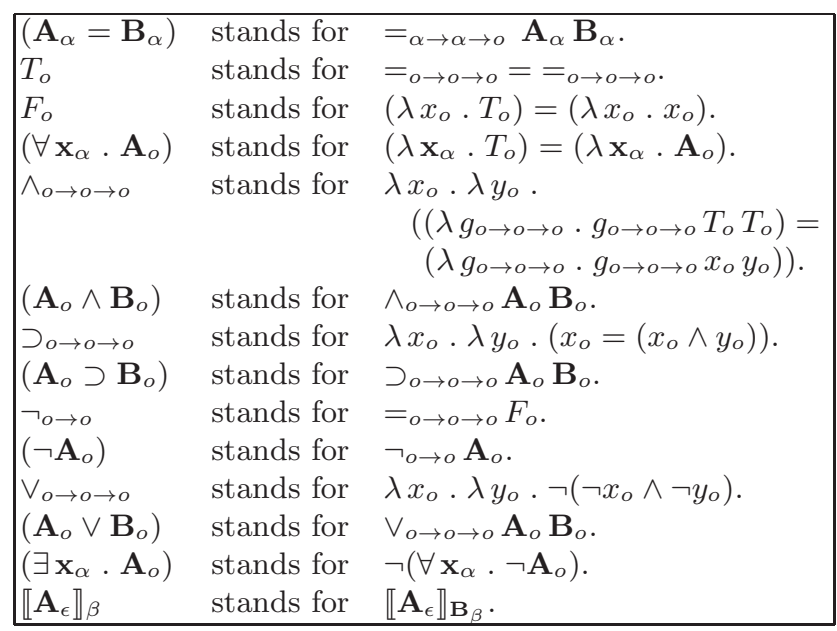

Table 3. Definitions and Abbreviations

\section{Semantics}

The semantics of $\mathrm{CTT}_{\mathrm{qe}}$ extends the semantics of $\mathcal{Q}_{0}$ [2] pp. 238-239] by defining the domain of the type $\epsilon$ and what quotations and evaluations mean.

\subsection{Frames}

A frame of $\mathrm{CTT}_{\mathrm{qe}}$ is a collection $\left\{D_{\alpha} \mid \alpha \in \mathcal{T}\right\}$ of domains such that:

1. $D_{\iota}$ is a nonempty set of values (called individuals).

2. $D_{o}=\{\mathrm{T}, \mathrm{F}\}$, the set of standard truth values.

3. $D_{\epsilon}$ is the set of constructions of $\mathrm{CTT}_{\mathrm{qe}}$.

4. For $\alpha, \beta \in \mathcal{T}, D_{\alpha \rightarrow \beta}$ is the set of total functions from $D_{\alpha}$ to $D_{\beta}$. 


\subsection{Interpretations}

An interpretation of $\mathrm{CTT}_{\mathrm{qe}}$ is a pair $\left(\left\{D_{\alpha} \mid \alpha \in \mathcal{T}\right\}, I\right)$ consisting of a frame and an interpretation function $I$ that maps each constant in $\mathcal{C}$ of type $\alpha$ to an element of $D_{\alpha}$ such that:

1. For all $\alpha \in \mathcal{T}, I\left(=_{\alpha \rightarrow \alpha \rightarrow o}\right)$ is the function $f \in D_{\alpha \rightarrow \alpha \rightarrow o}$ such that, for all $d_{1}, d_{2} \in D_{\alpha}, f\left(d_{1}\right)\left(d_{2}\right)=\mathrm{T}$ iff $d_{1}=d_{2}$. That is, $I\left(=_{\alpha \rightarrow \alpha \rightarrow o}\right)$ is the identity relation on $D_{\alpha}$.

2. $I$ (is- $\operatorname{var}_{\epsilon \rightarrow o}$ ) is the function $f \in D_{\epsilon \rightarrow o}$ such that, for all $\mathbf{A}_{\epsilon} \in D_{\epsilon}, f\left(\mathbf{A}_{\epsilon}\right)=\mathrm{T}$ iff $\mathbf{A}_{\epsilon}=\left\ulcorner\mathbf{x}_{\alpha}\right\urcorner$ for some variable $\mathbf{x}_{\alpha} \in \mathcal{V}$.

3. $I$ (is-con is $\rightarrow 0$ ) is the function $f \in D_{\epsilon \rightarrow o}$ such that, for all $\mathbf{A}_{\epsilon} \in D_{\epsilon}, f\left(\mathbf{A}_{\epsilon}\right)=\mathrm{T}$ iff $\mathbf{A}_{\epsilon}=\left\ulcorner\mathbf{c}_{\alpha}\right\urcorner$ for some constant $\mathbf{c}_{\alpha} \in \mathcal{C}$.

4. $I\left(\right.$ app $\left._{\epsilon \rightarrow \epsilon \rightarrow \epsilon}\right)$ is the function $f \in D_{\epsilon \rightarrow \epsilon \rightarrow \epsilon}$ such that, for all $\mathbf{A}_{\epsilon}, \mathbf{B}_{\epsilon} \in D_{\epsilon}$, $f\left(\mathbf{A}_{\epsilon}\right)\left(\mathbf{B}_{\epsilon}\right)$ is the construction app $\cot _{\epsilon \rightarrow \epsilon} \mathbf{A}_{\epsilon} \mathbf{B}_{\epsilon}$.

5. $I\left(\right.$ abs $\left._{\epsilon \rightarrow \epsilon \rightarrow \epsilon}\right)$ is the function $f \in D_{\epsilon \rightarrow \epsilon \rightarrow \epsilon}$ such that, for all $\mathbf{A}_{\epsilon}, \mathbf{B}_{\epsilon} \in D_{\epsilon}$, $f\left(\mathbf{A}_{\epsilon}\right)\left(\mathbf{B}_{\epsilon}\right)$ is the construction abs $_{\epsilon \rightarrow \epsilon \rightarrow \epsilon} \mathbf{A}_{\epsilon} \mathbf{B}_{\epsilon}$.

6. $I\left(\right.$ quo $\left._{\epsilon \rightarrow \epsilon}\right)$ is the function $f \in D_{\epsilon \rightarrow \epsilon}$ such that, for all $\mathbf{A}_{\epsilon} \in D_{\epsilon}, f\left(\mathbf{A}_{\epsilon}\right)$ is the construction quo ${ }_{\epsilon \rightarrow \epsilon} \mathbf{A}_{\epsilon}$.

7. For all $\alpha \in \mathcal{T}, I$ (is-expr ${ }_{\epsilon \rightarrow o}^{\alpha}$ ) is the function $f \in D_{\epsilon \rightarrow o}$ such that, for all $\mathbf{A}_{\epsilon} \in D_{\epsilon}, f\left(\mathbf{A}_{\epsilon}\right)=\mathrm{T}$ iff $\mathbf{A}_{\epsilon}=\mathcal{E}\left(\mathbf{B}_{\alpha}\right)$ for some (eval-free) expression $\mathbf{B}_{\alpha}$.

Remark 3.21 (Domain of Constructions) We would prefer to define $D_{\epsilon}$ to be the set of proper constructions because we need only proper constructions to represent the syntax trees of eval-free expressions. However, then the natural interpretations of the three syntax constructors - app $\operatorname{ap\rightarrow \epsilon \rightarrow \epsilon }_{\epsilon}$, abs $\operatorname{si\epsilon \rightarrow \epsilon }_{\epsilon \rightarrow}$, and quo $_{\epsilon \rightarrow \epsilon}$ - would be partial functions. Since $\mathrm{CTT}_{\mathrm{qe}}$ admits only total functions, it is more convenient to allow $D_{\epsilon}$ to include improper constructions than to interpret the syntax constructors as total functions that represent partial functions.

An assignment into a frame $\left\{D_{\alpha} \mid \alpha \in \mathcal{T}\right\}$ is a function $\varphi$ whose domain is $\mathcal{V}$ such that, for each variable $\mathbf{x}_{\alpha}, \varphi\left(\mathbf{x}_{\alpha}\right) \in D_{\alpha}$. Given an assignment $\varphi$, a variable $\mathbf{x}_{\alpha}$, and $d \in D_{\alpha}$, let $\varphi\left[\mathbf{x}_{\alpha} \mapsto d\right]$ be the assignment $\psi$ such that $\psi\left(\mathbf{x}_{\alpha}\right)=d$ and $\psi\left(\mathbf{y}_{\beta}\right)=\varphi\left(\mathbf{y}_{\beta}\right)$ for all variables $\mathbf{y}_{\beta} \neq \mathbf{x}_{\alpha}$. Given an interpretation $\mathcal{M}=\left(\left\{D_{\alpha} \mid \alpha \in \mathcal{T}\right\}, I\right)$, assign $(\mathcal{M})$ is the set of assignments into the frame of $\mathcal{M}$.

\subsection{Models}

An interpretation $\mathcal{M}=\left(\left\{D_{\alpha} \mid \alpha \in \mathcal{T}\right), I\right\}$ is a model for $\mathrm{CTT}_{\mathrm{qe}}$ if there is a binary valuation function $V^{\mathcal{M}}$ such that, for all assignments $\varphi \in \operatorname{assign}(\mathcal{M})$ and expressions $\mathbf{C}_{\gamma}, V_{\varphi}^{\mathcal{M}}\left(\mathbf{C}_{\gamma}\right) \in D_{\gamma}$ and each of the following conditions is satisfied:

1. If $\mathbf{C}_{\gamma} \in \mathcal{V}$, then $V_{\varphi}^{\mathcal{M}}\left(\mathbf{C}_{\gamma}\right)=\varphi\left(\mathbf{C}_{\gamma}\right)$.

2. If $\mathbf{C}_{\gamma} \in \mathcal{C}$, then $V_{\varphi}^{\mathcal{M}}\left(\mathbf{C}_{\gamma}\right)=I\left(\mathbf{C}_{\gamma}\right)$.

3. If $\mathbf{C}_{\gamma}$ is $\mathbf{F}_{\alpha \rightarrow \beta} \mathbf{A}_{\alpha}$, then $V_{\varphi}^{\mathcal{M}}\left(\mathbf{C}_{\gamma}\right)=V_{\varphi}^{\mathcal{M}}\left(\mathbf{F}_{\alpha \rightarrow \beta}\right)\left(V_{\varphi}^{\mathcal{M}}\left(\mathbf{A}_{\alpha}\right)\right)$. 
4. If $\mathbf{C}_{\gamma}$ is $\lambda \mathbf{x}_{\alpha} . \mathbf{B}_{\beta}$, then $V_{\varphi}^{\mathcal{M}}\left(\mathbf{C}_{\gamma}\right)$ is the function $f \in D_{\alpha \rightarrow \beta}$ such that, for each $d \in D_{\alpha}, f(d)=V_{\varphi\left[\mathbf{x}_{\alpha} \mapsto d\right]}^{\mathcal{M}}\left(\mathbf{B}_{\beta}\right)$.

5. If $\mathbf{C}_{\gamma}$ is $\left\ulcorner\mathbf{A}_{\alpha}\right\urcorner$, then $V_{\varphi}^{\mathcal{M}}\left(\mathbf{C}_{\gamma}\right)=\mathcal{E}\left(\mathbf{A}_{\alpha}\right)$.

6. If $\mathbf{C}_{\gamma}$ is $\llbracket \mathbf{A}_{\epsilon} \rrbracket_{\beta}$ and $V_{\varphi}^{\mathcal{M}}$ (is-expr $\left.\boldsymbol{i n o}_{\epsilon \rightarrow o}^{\beta} \mathbf{A}_{\epsilon}\right)=\mathrm{T}$, then

$$
V_{\varphi}^{\mathcal{M}}\left(\mathbf{C}_{\gamma}\right)=V_{\varphi}^{\mathcal{M}}\left(\mathcal{E}^{-1}\left(V_{\varphi}^{\mathcal{M}}\left(\mathbf{A}_{\epsilon}\right)\right)\right)
$$

Proposition 3.31 Models for $\mathrm{CTT}_{\mathrm{qe}}$ exist.

Proof. It is easy to construct an interpretation $\mathcal{M}=\left(\left\{\mathcal{D}_{\alpha} \mid \alpha \in \mathcal{T}\right\}, I\right)$ that is a model for $\mathrm{CTT}_{\mathrm{qe}}$. Note that, if $V_{\varphi}^{\mathcal{M}}\left(\right.$ is- $\left.^{-\operatorname{expr}_{\epsilon \rightarrow o}^{\beta}} \mathbf{A}_{\epsilon}\right)=\mathrm{F}$, then $V_{\varphi}^{\mathcal{M}}\left(\llbracket \mathbf{A}_{\epsilon} \rrbracket_{\beta}\right)$ can be any value in $D_{\beta}$.

Remark 3.32 (Standard vs. General Models) The notion of a model defined here is a standard model in which each function domain $D_{\alpha \rightarrow \beta}$ is the set of all total functions from $D_{\alpha}$ to $D_{\beta}$. Andrews' semantics for $\mathcal{Q}_{0}$ is based on the notion of a general model, introduced by Leon Henkin [15, in which each function domain $D_{\alpha \rightarrow \beta}$ is a set of some total functions from $D_{\alpha}$ to $D_{\beta}$. General models can be easily defined for $\mathrm{CTT}_{\mathrm{qe}}$. The definition of a frame, however, has to be changed so that the domain $D_{\epsilon}$ may include "nonstandard constructions".

Remark 3.33 (Semantics of Evaluations) When $V_{\varphi}^{\mathcal{M}}\left(\right.$ is-expr $\left.{ }_{\epsilon \rightarrow o}^{\beta} \mathbf{A}_{\epsilon}\right)=\mathrm{T}$, the semantics of $V_{\varphi}^{\mathcal{M}}\left(\llbracket \mathbf{A}_{\epsilon} \rrbracket_{\beta}\right)$ involves a double valuation as mentioned in the Double Substitution Problem described in the Introduction.

Remark 3.34 (Undefined Evaluations) Suppose $V_{\varphi}^{\mathcal{M}}\left(\mathbf{A}_{\epsilon}\right)$ is an improper construction. Then $V_{\varphi}^{\mathcal{M}}\left(\mathcal{E}^{-1}\left(V_{\varphi}^{\mathcal{M}}\left(\mathbf{A}_{\epsilon}\right)\right)\right)$ is undefined and $V_{\varphi}^{\mathcal{M}}\left(\llbracket \mathbf{A}_{\epsilon} \rrbracket_{\beta}\right)$ has no natural value. Since $\mathrm{CTT}_{\mathrm{qe}}$ does not admit undefined expressions, $V_{\varphi}^{\mathcal{M}}\left(\llbracket \mathbf{A}_{\epsilon} \rrbracket_{\beta}\right)$ is defined but its value is unspecified. Similarly, if $V_{\varphi}^{\mathcal{M}}\left(\mathbf{A}_{\epsilon}\right)$ is a proper construction of the form $\mathcal{E}\left(\mathbf{B}_{\gamma}\right)$ with $\gamma \neq \beta, V_{\varphi}^{\mathcal{M}}\left(\llbracket \mathbf{A}_{\epsilon} \rrbracket_{\beta}\right)$ is unspecified.

Let $\mathcal{M}$ be a model for $\mathrm{CTT}_{\mathrm{qe}}$. $\mathbf{A}_{o}$ is valid in $\mathcal{M}$, written $\mathcal{M} \models \mathbf{A}_{o}$, if $\mathcal{V}_{\varphi}^{\mathcal{M}}\left(\mathbf{A}_{o}\right)=\mathrm{T}$ for all assignments $\varphi \in \operatorname{assign}(\mathcal{M})$.

Proposition 3.35 Let $\mathcal{M}$ be a model for $\mathrm{CTT}_{\mathrm{qe}}, \boldsymbol{A}_{\epsilon}$ be a construction, and $\varphi \in \operatorname{assign}(\mathcal{M})$. Then $\mathcal{V}_{\varphi}^{\mathcal{M}}\left(\boldsymbol{A}_{\epsilon}\right)=\boldsymbol{A}_{\epsilon}$.

Proof. Follows immediately from conditions $4-6$ of the definition of an interpretation and condition 5 of the definition of a model.

Theorem 3.36 (Law of Quotation) $\left\ulcorner\boldsymbol{A}_{\alpha}\right\urcorner=\mathcal{E}\left(\boldsymbol{A}_{\alpha}\right)$ is valid in every model of $\mathrm{CTT}_{\mathrm{qe}}$.

Proof. Let $\mathcal{M}$ be a model of $\operatorname{CTT}_{\mathrm{qe}}$ and $\varphi \in \operatorname{assign}(\mathcal{M})$. Then

$$
\begin{aligned}
& \mathcal{V}_{\varphi}^{\mathcal{M}}\left(\left\ulcorner\mathbf{A}_{\alpha}\right\urcorner\right) \\
& =\mathcal{E}\left(\mathbf{A}_{\alpha}\right) \\
& =\mathcal{V}_{\varphi}^{\mathcal{M}}\left(\mathcal{E}\left(\mathbf{A}_{\alpha}\right)\right)
\end{aligned}
$$


(2) follows from condition 5 of the definition of a model, and (3) follows from Proposition 3.35. Hence $\mathcal{V}_{\varphi}^{\mathcal{M}}\left(\left\ulcorner\mathbf{A}_{\alpha}\right\urcorner\right)=V_{\varphi}^{\mathcal{M}}\left(\mathcal{E}\left(\mathbf{A}_{\alpha}\right)\right)$ for all $\varphi \in \operatorname{assign}(\mathcal{M})$ which implies $\left\ulcorner\mathbf{A}_{\alpha}\right\urcorner=\mathcal{E}\left(\mathbf{A}_{\alpha}\right)$ is valid in $\mathcal{M}$.

Theorem 3.37 (Law of Disquotation) $\llbracket\left\ulcorner\boldsymbol{A}_{\alpha}\right\urcorner \rrbracket_{\alpha}=\boldsymbol{A}_{\alpha}$ is valid in every model of $\mathrm{CTT}_{\mathrm{qe}}$.

Proof. Let $\mathcal{M}$ be a model of $\operatorname{CTT}_{\mathrm{qe}}$ and $\varphi \in \operatorname{assign}(\mathcal{M})$. Then

$$
\begin{aligned}
& \mathcal{V}_{\varphi}^{\mathcal{M}}\left(\llbracket\left\ulcorner\mathbf{A}_{\alpha}\right\urcorner \rrbracket_{\alpha}\right) \\
& =\mathcal{V}_{\varphi}^{\mathcal{M}}\left(\mathcal{E}^{-1}\left(\mathcal{V}_{\varphi}^{\mathcal{M}}\left(\left\ulcorner\mathbf{A}_{\alpha}\right\urcorner\right)\right)\right) \\
& =\mathcal{V}_{\varphi}^{\mathcal{M}}\left(\mathcal{E}^{-1}\left(\mathcal{E}\left(\mathbf{A}_{\alpha}\right)\right)\right) \\
& =\mathcal{V}_{\varphi}^{\mathcal{M}}\left(\mathbf{A}_{\alpha}\right)
\end{aligned}
$$

Since $V_{\varphi}^{\mathcal{M}}\left(\right.$ is-expr $\left.\operatorname{ex\rightarrow o}_{\epsilon \rightarrow}^{\alpha}\left\ulcorner\mathbf{A}_{\alpha}\right\urcorner\right)=\mathrm{T}$, (2) follows from condition 6 of the definition of a model. $\mathcal{V}_{\varphi}^{\mathcal{M}}\left(\left\ulcorner\mathbf{A}_{\alpha}\right\urcorner\right)=\mathcal{E}\left(\mathbf{A}_{\alpha}\right)$ by condition 5 of the definition of a model. (3) and (4) are then immediate. Hence $\mathcal{V}_{\varphi}^{\mathcal{M}}\left(\llbracket\left\ulcorner\mathbf{A}_{\alpha}\right\urcorner \rrbracket_{\alpha}\right)=\mathcal{V}_{\varphi}^{\mathcal{M}}\left(\mathbf{A}_{\alpha}\right)$ for all $\varphi \in \operatorname{assign}(\mathcal{M})$ which implies $\llbracket\left\ulcorner\mathbf{A}_{\alpha}\right\urcorner \rrbracket_{\alpha}=\mathbf{A}_{\alpha}$ is valid in $\mathcal{M}$.

Remark 3.38 (Evaluation Problem) Theorem 3.37 shows that disquotation holds universally in $\mathrm{CTT}_{\mathrm{qe}}$ contrary to the Evaluation Problem described in the Introduction. We have avoided the Evaluation Problem in $\mathrm{CTT}_{\mathrm{qe}}$ by admitting only quotations of eval-free expressions. If quotations of non-eval-free expressions were allowed in $\mathrm{CTT}_{\mathrm{qe}}$, the logic would be significantly more expressive, but also much more complicated, as seen in $\mathcal{Q}_{0}^{\mathrm{uqe}}[9$.

Remark 3.39 (Quotation restricted to Closed Expressions) If quotation is restricted to closed eval-free expressions in $\mathrm{CTT}_{\mathrm{qe}}$, then the Variable Problem and Double Substitution Problem disappear. However, most of the usefulness of having quotation and evaluation in $\mathrm{CTT}_{\mathrm{qe}}$ would also disappear which is illustrated by the examples in the next section.

\section{Examples}

We will present in this section four examples that illustrate the utility of the quotation and evaluation facility in $\mathrm{CTT}_{\mathrm{qe}}$.

\subsection{Reasoning about Syntax}

Reasoning about the syntax of expressions is normally performed in the metalogic, but in $\mathrm{CTT}_{\mathrm{qe}}$ reasoning about the syntax of eval-free expressions can be performed in the logic itself. This is done by reasoning about constructions (which represent the syntax trees of eval-free expressions) using quotation and the machinery of constructions. Algorithms that manipulate eval-free expressions 
can be formalized as functions that manipulate constructions. The functions can be executed using beta-reduction, rewriting, and other kinds of simplification.

As an example, consider the constant make-implication $\operatorname{tat\epsilon \rightarrow \epsilon }_{\epsilon}$ defined as

$$
\lambda x_{\epsilon} \cdot \lambda y_{\epsilon} \cdot\left(\operatorname{app}_{\epsilon \rightarrow \epsilon \rightarrow \epsilon}\left(\operatorname{app}_{\epsilon \rightarrow \epsilon \rightarrow \epsilon}\left\ulcorner\supset_{o \rightarrow o \rightarrow o}\right\urcorner x_{\epsilon}\right) y_{\epsilon}\right) .
$$

It can be used to build constructions that represent implications. As another example, consider the constant is-app $\cos _{\epsilon \rightarrow}$ defined as

$$
\lambda x_{\epsilon} \cdot \exists y_{\epsilon} \cdot \exists z_{\epsilon} \cdot x_{\epsilon}=\left(\operatorname{app}_{\epsilon \rightarrow \epsilon \rightarrow \epsilon} y_{\epsilon} z_{\epsilon}\right) .
$$

It can be used to test whether a construction represents a function application.

Reasoning about syntax is a two-step process: First, a construction is built using quotation and the machinery of constructions, and second, the construction is employed using evaluation. Continuing the example above,

$$
\text { make-implication }_{\epsilon \rightarrow \epsilon \rightarrow \epsilon}\left\ulcorner\mathbf{A}_{o}\right\urcorner\left\ulcorner\mathbf{B}_{o}\right\urcorner
$$

builds a construction equivalent to the quotation $\left\ulcorner\mathbf{A}_{o} \supset \mathbf{B}_{o}\right\urcorner$ and

$$
\text { 【make-implication } \text { m } \rightarrow \epsilon \rightarrow \epsilon\left\ulcorner\mathbf{A}_{o}\right\urcorner\left\ulcorner\mathbf{B}_{o}\right\urcorner \rrbracket_{o}
$$

employs the construction as the implication $\mathbf{A}_{o} \supset \mathbf{B}_{o}$. Using this mixture of quotation and evaluation, it is possible to express the interplay of syntax and semantics that is needed to formalize syntax-based algorithms that are commonly used in mathematics 8 . See section 4.4 for an example.

\subsection{Quasiquotation}

Quasiquotation is a parameterized form of quotation in which the parameters serve as holes in a quotation that are filled with expressions that denote syntactic values. It is a very powerful syntactic device for specifying expressions and defining macros. Quasiquotation was introduced by Willard Van Orman Quine in 1940 in the first version of his book Mathematical Logic [19]. It has been extensively employed in the Lisp family of programming languages [3, 4

In $\mathrm{CTT}_{\mathrm{qe}}$, constructing a large quotation from smaller quotations can be tedious because it requires many applications of syntax constructors. Quasiquotation provides a convenient way to construct big quotations from little quotations. It can be defined straightforwardly in $\mathrm{CTT}_{\mathrm{qe}}$.

A quasi-expression of $\mathrm{CTT}_{\mathrm{qe}}$ is defined inductively as follows:

1. $\left\lfloor\mathbf{A}_{\epsilon}\right\rfloor$ is a quasi-expression called an antiquotation.

2. $\mathbf{x}_{\alpha}$ is a quasi-expression.

3. $\mathbf{c}_{\alpha}$ is a quasi-expression.

${ }^{4}$ In Lisp, the standard symbol for quasiquotation is the backquote (") symbol, and thus in Lisp, quasiquotation is usually called backquote. 
4. If $M$ and $N$ are quasi-expressions, then $(M N),\left(\lambda \mathbf{x}_{\alpha} \cdot N\right),\left(\lambda\left\lfloor\mathbf{A}_{\epsilon}\right\rfloor . N\right)$, and $\ulcorner M\urcorner$ are quasi-expressions.

A quasi-expression is thus an expression where one or more subexpressions have been replaced by antiquotations. For example, $\neg\left(\mathbf{A}_{o} \wedge\left\lfloor\mathbf{B}_{\epsilon}\right\rfloor\right)$ is a quasi-expression. Obviously, every expression is a quasi-expression.

Let $\mathcal{E}^{\prime}$ be the function mapping quasi-expressions to expressions of type $\epsilon$ that is defined inductively as follows:

1. $\mathcal{E}^{\prime}\left(\left\lfloor\mathbf{A}_{\epsilon}\right\rfloor\right)=\mathbf{A}_{\epsilon}$.

2. $\mathcal{E}^{\prime}\left(\mathbf{x}_{\alpha}\right)=\left\ulcorner\mathbf{x}_{\alpha}\right\urcorner$.

3. $\mathcal{E}^{\prime}\left(\mathbf{c}_{\alpha}\right)=\left\ulcorner\mathbf{c}_{\alpha}\right\urcorner$.

4. $\mathcal{E}^{\prime}(M N)=\operatorname{app}_{\epsilon \rightarrow \epsilon \rightarrow \epsilon} \mathcal{E}^{\prime}(M) \mathcal{E}^{\prime}(N)$.

5. $\mathcal{E}^{\prime}(\lambda M . N)=\operatorname{abs}_{\epsilon \rightarrow \epsilon \rightarrow \epsilon} \mathcal{E}^{\prime}(M) \mathcal{E}^{\prime}(N)$.

6. $\mathcal{E}(\ulcorner M\urcorner)=$ quo $_{\epsilon \rightarrow \epsilon} \mathcal{E}^{\prime}(M)$.

Notice that $\mathcal{E}^{\prime}(M)=\mathcal{E}(M)$ when $M$ is an expression. Continuing our example above, $\mathcal{E}^{\prime}\left(\neg\left(\mathbf{A}_{o} \wedge\left\lfloor\mathbf{B}_{\epsilon}\right\rfloor\right)\right)=$

$$
\operatorname{app}_{\epsilon \rightarrow \epsilon \rightarrow \epsilon}\left\ulcorner\neg_{o \rightarrow o}\right\urcorner\left(\operatorname{app}_{\epsilon \rightarrow \epsilon \rightarrow \epsilon}\left(\operatorname{app}_{\epsilon \rightarrow \epsilon \rightarrow \epsilon}\left\ulcorner\wedge_{o \rightarrow o \rightarrow o}\right\urcorner \mathcal{E}^{\prime}\left(\mathbf{A}_{o}\right)\right) \mathbf{B}_{\epsilon}\right) .
$$

A quasiquotation is an expression of the form $\ulcorner M\urcorner$ where $M$ is a quasiexpression. Thus every quotation is a quasiquotation. The quasiquotation $\ulcorner M\urcorner$ serves as an alternate notation for the expression $\mathcal{E}^{\prime}(M)$. So $\left\ulcorner\neg\left(\mathbf{A}_{o} \wedge\left\lfloor\mathbf{B}_{\epsilon}\right\rfloor\right)\right\urcorner$ stands for the significantly more verbose expression in the previous paragraph. It represents the syntax tree of a negated conjunction in which the part of the tree corresponding to the second conjunct is replaced by the syntax tree represented by $\mathbf{B}_{\epsilon}$. If $\mathbf{B}_{\epsilon}$ is a quotation $\left\ulcorner\mathbf{C}_{o}\right\urcorner$, then the quasiquotation $\left\ulcorner\neg\left(\mathbf{A}_{o} \wedge\left\lfloor\left\ulcorner\mathbf{C}_{o}\right\urcorner\right\rfloor\right)\right\urcorner$ is equivalent to the quotation $\left\ulcorner\neg\left(\mathbf{A}_{o} \wedge \mathbf{C}_{o}\right)\right\urcorner$.

\subsection{Schemas}

A schema is a metalogical expression containing syntactic variables. An instance of a schema is a logical expression obtained by replacing the syntactic variables with appropriate logical expressions. In $\mathrm{CTT}_{\mathrm{qe}}$, a schema can be formalized as a single logical expression.

For example, consider the law of excluded middle (LEM) that is expressed as the formula schema $A \vee \neg A$ where $A$ is a syntactic variable ranging over all formulas. LEM can be formalized in $\mathrm{CTT}_{\mathrm{qe}}$ as the universal statement

$$
\forall x_{\epsilon} . \text { is- } \operatorname{expr}_{\epsilon \rightarrow o}^{o} x_{\epsilon} \supset \llbracket x_{\epsilon} \rrbracket_{o} \vee \neg \llbracket x_{\epsilon} \rrbracket_{o} .
$$

An instance of this formalization of LEM is any instance of the universal statement. Using quasiquotation, LEM could also be formalized in $\mathrm{CTT}_{\mathrm{qe}}$ as

$$
\forall x_{\epsilon} . \text { is- } \operatorname{expr}_{\epsilon \rightarrow o}^{o} x_{\epsilon} \supset \llbracket\left\ulcorner\left\lfloor x_{\epsilon}\right\rfloor \vee \neg\left\lfloor x_{\epsilon}\right\rfloor\right\urcorner \rrbracket_{o} .
$$


If we assume that the domain of the type $\iota$ is the natural numbers and $\mathcal{C}$ includes the usual constants of natural number arithmetic (including a constant $\mathrm{S}_{\iota \rightarrow \iota}$ representing the successor function), then the (first-order) induction schema for Peano arithmetic can be formalized in $\mathrm{CTT}_{\mathrm{qe}}$ as

$$
\begin{aligned}
& \forall f_{\epsilon} \cdot\left(\text { is- }^{-\operatorname{expr}_{\epsilon \rightarrow o}^{\iota \rightarrow o}} f_{\epsilon} \wedge \text { is-peano }_{\epsilon \rightarrow o} f_{\epsilon}\right) \supset \\
& \quad\left(\left(\llbracket f_{\epsilon} \rrbracket_{\iota \rightarrow o} 0\right.\right.\left.\left.\wedge\left(\forall x_{\iota} \cdot \llbracket f_{\epsilon} \rrbracket_{\iota \rightarrow o} x_{\iota} \supset \llbracket f_{\epsilon} \rrbracket_{\iota \rightarrow o}\left(\mathrm{~S}_{\iota \rightarrow \iota} x_{\iota}\right)\right)\right) \supset \forall x_{\iota} \cdot \llbracket f_{\epsilon} \rrbracket_{\iota \rightarrow o} x_{\iota}\right)
\end{aligned}
$$

where is-peano $\rightarrow_{\epsilon \rightarrow o} f_{\epsilon}$ holds iff $f_{\epsilon}$ represents the syntactic tree of a formula of first-order Peano arithmetic. Hence it is possible to directly formalize first-order Peano arithmetic in $\mathrm{CTT}_{\mathrm{qe}}$. The induction schema for Presburger arithmetic can be formalized similarly using an appropriate predicate is-presburger pr $_{\epsilon \rightarrow 0}$.

\subsection{Meaning Formulas}

Many symbolic algorithms work by manipulating mathematical expressions in a mathematically meaningful way. A meaning formula for such an algorithm is a statement that captures the mathematical relationship between the input and output expressions of the algorithm. For example, consider a symbolic differentiation algorithm that takes as input an expression (say $x^{2}$ ), repeatedly applies syntactic differentiation rules to the expression, and then returns as output the final expression $(2 x)$ that is produced. The intended meaning formula of this algorithm states that the function $(\lambda x: \mathbb{R} .2 x)$ represented by the output expression is the derivative of the function $\left(\lambda x: \mathbb{R} . x^{2}\right)$ represented by the input expression.

Meaning formulas are difficult to express in a traditional logic like first-order logic or simple type theory since there is no way to directly refer to the syntactic structure of the expressions in the logic [8]. However, meaning formulas can be easily expressed in $\mathrm{CTT}_{\mathrm{qe}}$.

Consider the following example. Assume that the domain of the type $\iota$ is the real numbers and $\mathcal{C}$ includes the usual constants of real number arithmetic plus (1) is-poly plo $_{\epsilon \rightarrow o}$ such that is-poly ${ }_{\epsilon \rightarrow o} \mathbf{A}_{\epsilon}=\mathrm{T}$ iff $\mathbf{A}_{\epsilon}$ represents a syntax tree of an expression of type $\iota$ that is a polynomial, (2) $\operatorname{deriv}_{(\iota \rightarrow \iota) \rightarrow(\iota \rightarrow \iota)}$ such that deriv $(\iota \rightarrow \iota) \rightarrow(\iota \rightarrow \iota) \mathbf{F}_{\iota \rightarrow \iota}$ is the derivative of the function $\mathbf{F}_{\iota \rightarrow \iota}$, and (3) poly-diff $_{\epsilon \rightarrow \epsilon \rightarrow \epsilon}$ such that, if is-poly ${ }_{\epsilon \rightarrow o} \mathbf{A}_{\epsilon}$ holds, then poly-diff ${ }_{\epsilon \rightarrow \epsilon \rightarrow \epsilon} \mathbf{A}_{\epsilon}\left\ulcorner\mathbf{x}_{\iota}\right\urcorner$ is the result of applying the usual differentiation rules for polynomials to $\mathbf{A}_{\epsilon}$ with respect to $\mathbf{x}_{\iota}$. Then the meaning formula for poly-diff ${ }_{\epsilon \rightarrow \epsilon \rightarrow \epsilon}$ is

$$
\begin{aligned}
\forall u_{\epsilon} & \forall v_{\epsilon} \cdot\left(\text { is- } \operatorname{var}_{\epsilon \rightarrow o} u_{\epsilon} \wedge \text { is-expr }_{\epsilon \rightarrow o}^{\iota} u_{\epsilon} \wedge \text { is-poly }_{\epsilon \rightarrow o} v_{\epsilon}\right) \supset \\
& \operatorname{deriv}_{(\iota \rightarrow \iota) \rightarrow(\iota \rightarrow \iota)}\left(\llbracket \text { abs }_{\epsilon \rightarrow \epsilon \rightarrow \epsilon} u_{\epsilon} v_{\epsilon} \rrbracket_{\iota \rightarrow \iota}\right)= \\
& \llbracket \text { abss }_{\epsilon \rightarrow \epsilon \rightarrow \epsilon} u_{\epsilon}(\text { poly-diff } \\
&
\end{aligned}
$$

\footnotetext{
${ }^{5}$ We restrict this example to polynomials since polynomial functions and their derivatives are always total. Thus issues of undefinedness do not arise in the formulation of the meaning formula for poly-diff
} 
The string of equations

$$
\begin{aligned}
& \operatorname{deriv}_{(\iota \rightarrow \iota) \rightarrow(\iota \rightarrow \iota)}\left(\lambda x_{\iota} \cdot x_{\iota}^{2}\right) \\
& =\operatorname{deriv}_{(\iota \rightarrow \iota) \rightarrow(\iota \rightarrow \iota)}\left(\llbracket\left\ulcorner\lambda x_{\iota} \cdot x_{\iota}^{2}\right\urcorner \rrbracket_{\iota \rightarrow \iota}\right) \\
& =\operatorname{deriv}_{(\iota \rightarrow \iota) \rightarrow(\iota \rightarrow \iota)}\left(\llbracket \operatorname{abs}_{\epsilon \rightarrow \epsilon \rightarrow \epsilon}\left\ulcorner x_{\iota}\right\urcorner\left\ulcorner x_{\iota}^{2}\right\urcorner \rrbracket_{\iota \rightarrow \iota}\right) \\
& =\llbracket \operatorname{abs}_{\epsilon \rightarrow \epsilon \rightarrow \epsilon}\left\ulcorner x_{\iota}\right\urcorner\left(\text { poly-diff }_{\epsilon \rightarrow \epsilon \rightarrow \epsilon}\left\ulcorner x_{\iota}^{2}\right\urcorner\left\ulcorner x_{\iota}\right\urcorner\right) \rrbracket_{\iota \rightarrow \iota} \\
& =\llbracket \operatorname{abs}_{\epsilon \rightarrow \epsilon \rightarrow \epsilon}\left\ulcorner x_{\iota}\right\urcorner\left\ulcorner 2 * x_{\iota}\right\urcorner \rrbracket_{\iota \rightarrow \iota} \\
& =\llbracket\left\ulcorner\lambda x_{\iota} \cdot 2 * x_{\iota}\right\urcorner \rrbracket_{\iota \rightarrow \iota} \\
& =\lambda x_{\iota} \cdot 2 * x_{\iota}
\end{aligned}
$$

proves (informally) the desired result where the equation given by (3) and (4) results from instantiating the meaning formula for poly-diff ${ }_{\epsilon \rightarrow \epsilon \rightarrow \epsilon}$ with $\left\ulcorner x_{\iota}\right\urcorner$ and $\left\ulcorner x_{\iota}^{2}\right\urcorner$.

\section{A Sketch of a Simple Proof System}

At first glance, it would appear that a proof system for $\mathrm{CTT}_{\mathrm{qe}}$ could be straightforwardly developed by extending Andrews' proof system for $\mathcal{Q}_{0}$ [2, p. 213]. We can define is- $\operatorname{var}_{\epsilon \rightarrow o}$ (and is-con $\cos _{\epsilon \rightarrow o}$ in a similar way) by the axiom schemas is- $\operatorname{var}_{\epsilon \rightarrow o}\left\ulcorner\mathbf{x}_{\alpha}\right\urcorner$ and $\neg$ is-var $\operatorname{vato}_{\epsilon \rightarrow} \mathbf{A}_{\epsilon}$ where $\mathbf{A}_{\epsilon}$ is any construction that is not a quoted variable. We can recursively define is-expr ${ }_{\epsilon \rightarrow o}^{\alpha}$ using a set of axioms that say how expressions are constructed. We can specify that the type $\epsilon$ of constructions is an inductive type using a set of axioms that say (1) the constructions are distinct from each other and (2) induction holds for constructions. We can specify quotation using the Law of Quotation $\left\ulcorner\mathbf{A}_{\alpha}\right\urcorner=\mathcal{E}\left(\mathbf{A}_{\alpha}\right)$ (Theorem 3.36). And we can specify evaluation using the Law of Disquotation $\llbracket\left\ulcorner\mathbf{A}_{\alpha}\right\urcorner \rrbracket_{\alpha}=\mathbf{A}_{\alpha}$ (Theorem 3.37).

Andrews' proof system with these added axioms would enable simple theorems involving quotation and evaluation to be proved, but the proof system would not be able to substitute expressions for free variables occurring in the argument of an evaluation. Hence schemas and meaning formulas could be expressed in $\mathrm{CTT}_{\mathrm{qe}}$, but they would be useless because they could not be instantiated. Clearly, a useful proof system for $\mathrm{CTT}_{\mathrm{qe}}$ requires some form of substitution that is applicable to evaluations.

Due to the Variable Problem, substitution involving evaluations cannot be purely syntactic as in a traditional logic. It must be a semantics-dependent operation in which side conditions, like whether a variable is free in an expression, are proved within the proof system. Since $\mathrm{CTT}_{\mathrm{qe}}$ supports reasoning about syntax, an obvious way forward is to add to $\mathcal{C}$ a logical constant $\operatorname{sub}_{\epsilon \rightarrow \epsilon \rightarrow \epsilon \rightarrow \epsilon}$ such that, if $\mathbf{C}_{\beta}$ is the result of substituting $\mathbf{A}_{\alpha}$ for each free occurrence of $\mathbf{x}_{\alpha}$ in $\mathbf{B}_{\beta}$ without any variable captures, then

$$
\operatorname{sub}_{\epsilon \rightarrow \epsilon \rightarrow \epsilon \rightarrow \epsilon}\left\ulcorner\mathbf{A}_{\alpha}\right\urcorner\left\ulcorner\mathbf{x}_{\alpha}\right\urcorner\left\ulcorner\mathbf{B}_{\beta}\right\urcorner=\left\ulcorner\mathbf{C}_{\beta}\right\urcorner .
$$

$\operatorname{sub}_{\epsilon \rightarrow \epsilon \rightarrow \epsilon \rightarrow \epsilon}$ thus plays the role of an explicit substitution operator [1]. 
This approach, however, does not work in $\mathrm{CTT}_{\mathrm{qe}}$ since $\mathbf{B}_{\beta}$ may contain evaluations, but quotations in $\mathrm{CTT}_{\mathrm{qe}}$ may not contain evaluations. Although the approach does work in $\mathcal{Q}_{0}^{\text {uqe }}$ 9] in which quotations in СTT $_{\text {qe }}$ may contain evaluations, it is extremely complicated due to the Evaluation Problem.

A more promising approach is to add some axiom schemas to the five betareduction axiom schemas used by Andrews' in his proof system for $\mathcal{Q}_{0}$ [2, p. 213] that specify beta-reduction of an application of the form $\left(\lambda \mathbf{x}_{\alpha} \cdot \llbracket \mathbf{B}_{\epsilon} \rrbracket_{\beta}\right) \mathbf{A}_{\alpha}$. But how do we overcome the Double Substitution Problem? There seems to be no easy way of emulating a double substitution with beta-reduction, so the best approach appears to be to consider only cases that do not require a second substitution, as formalized by the following axiom schema:

$$
\begin{aligned}
& \left(\text { is-expr } \mathrm{ex}_{\epsilon \rightarrow o}^{\beta}\left(\left(\lambda \mathbf{x}_{\alpha} \cdot \mathbf{B}_{\epsilon}\right) \mathbf{A}_{\alpha}\right) \wedge \neg\left(\text { is-free-in } \mathrm{f}_{\epsilon \rightarrow \epsilon \rightarrow o}\left\ulcorner\mathbf{x}_{\alpha}\right\urcorner\left(\left(\lambda \mathbf{x}_{\alpha} \cdot \mathbf{B}_{\epsilon}\right) \mathbf{A}_{\alpha}\right)\right)\right) \supset \\
& \left(\lambda \mathbf{x}_{\alpha} \cdot \llbracket \mathbf{B}_{\epsilon} \rrbracket_{\beta}\right) \mathbf{A}_{\alpha}=\llbracket\left(\lambda \mathbf{x}_{\alpha} \cdot \mathbf{B}_{\epsilon}\right) \mathbf{A}_{\alpha} \rrbracket_{\beta} .
\end{aligned}
$$

Here is-free-in $\mathrm{in}_{\epsilon \rightarrow \epsilon \rightarrow o}$ would be a new logical constant in $\mathcal{C}$, and the second condition would say that $\mathbf{x}_{\alpha}$ is not free in the expression whose syntax tree is represented by $\left(\lambda \mathbf{x}_{\alpha} \cdot \mathbf{B}_{\epsilon}\right) \mathbf{A}_{\alpha}$. As a result, there would be no free occurrences of $\mathbf{x}_{\alpha}$ in the right-hand side of the conclusion after the evaluation is eliminated. Details of this approach will be given in a future paper that presents the proof system for $\mathrm{CTT}_{\mathrm{qe}}$ that we have sketched.

\section{Conclusion}

Quotation and evaluation provide a basis for metaprogramming as seen in Lisp and other programming languages. We believe that these mechanisms can also provide a basis for metareasoning in traditional logics like first-order logic or simple type theory. However, incorporating quotation and evaluation into a traditional logic is much more challenging than incorporating them into a programming language due to the three problems we described in the Introduction.

In this paper we have introduced $\mathrm{CTT}_{\mathrm{qe}}$, a logic based on $\mathcal{Q}_{0}$ [2], Andrews' version of Church's type theory, that includes quotation and evaluation. We have presented the syntax and semantics of $\mathrm{CTT}_{\mathrm{qe}}$, sketched a proof system for it, and given examples that show the practical benefit of having quotation and evaluation in a logic.

CTT $_{\mathrm{qe}}$ is a simpler version of $\mathcal{Q}_{0}^{\text {uqe }} \underline{9}$, a richer, but more complicated, version of $\mathcal{Q}_{0}$ with undefinedness, quotation, and evaluation. In $\mathcal{Q}_{0}^{\text {uqe }}$, quotation may be applied to expressions containing evaluations, expressions may be undefined and functions may be partial, and substitution is implemented explicitly as a logical constant. Allowing quotation to be applied to all expressions makes $\mathcal{Q}_{0}^{\text {uqe }}$ much more expressive than $\mathrm{CTT}_{\mathrm{qe}}$ but also much more difficult to implement since substitution in the presence of evaluations is highly complex. We believe that $\mathrm{CTT}_{\mathrm{qe}}$ would not be hard to implement. Since it is a version of Church's type theory, it could be implemented by extending an implementation of HOL [11] such as HOL Light [14. 
Our approach for incorporating quotation and evaluation into Church's type theory - introducing an inductive type of constructions, a quotation operator, and a typed evaluation operator - can be applied to other logics including many-sorted first-order logic. We have shown that developing the needed syntax and semantics is relatively straightforward, while developing a proof system for the logic is fraught with difficulties.

\section{Acknowledgments}

The author thanks the reviewers for their helpful comments and suggestions.

\section{References}

1. M. Abadi, L. Cardelli, P.-L. Curien, and J.-J. Lévy. Explicit substitution. Journal of Functional Programming, 1:375-416, 1991.

2. P. B. Andrews. An Introduction to Mathematical Logic and Type Theory: To Truth through Proof, Second Edition. Kluwer, 2002.

3. A. Bawden. Quasiquotation in Lisp. In O. Danvy, editor, Proceedings of the 1999 ACM SIGPLAN Symposium on Partial Evaluation and Semantics-Based Program Manipulation, pages 4-12, 1999. Technical report BRICS-NS-99-1, University of Aarhus, 1999.

4. A. Chlipala. Certified Programming with Dependent Types: A Pragmatic Introduction to the Coq Proof Assistant. MIT Press, 2013.

5. A. Church. A formulation of the simple theory of types. Journal of Symbolic Logic, $5: 56-68,1940$.

6. S. Costantini. Meta-reasoning: A survey. In A. C. Kakas and F. Sadri, editors, Computational Logic: Logic Programming and Beyond, Essays in Honour of Robert A. Kowalski, Part II, volume 2408 of Lecture Notes in Computer Science, pages 253-288, 2002.

7. W. M. Farmer. Chiron: A set theory with types, undefinedness, quotation, and evaluation. Computing Research Repository, abs/1305.6206 (154 pp.), 2013.

8. W. M. Farmer. The formalization of syntax-based mathematical algorithms using quotation and evaluation. In J. Carette, D. Aspinall, C. Lange, P. Sojka, and W. Windsteiger, editors, Intelligent Computer Mathematics, volume 7961 of Lecture Notes in Computer Science, pages 35-50. Springer, 2013.

9. W. M. Farmer. Simple type theory with undefinedness, quotation, and evaluation. Computing Research Repository, abs/1406.6706 (87 pp.), 2014.

10. G. Gonthier, A. Mahboubi, and E. Tassi. A Small Scale Reflection Extension for the Coq system. Research Report RR-6455, Inria Saclay Ile de France, 2015.

11. M. J. C. Gordon and T. F. Melham. Introduction to HOL: A Theorem Proving Environment for Higher Order Logic. Cambridge University Press, 1993.

12. J. Grundy, T. Melham, and J. O'Leary. A reflective functional language for hardware design and theorem proving. Journal of Functional Programming, 16, 2006.

13. J. Harrison. Metatheory and reflection in theorem proving: A survey and critique. Technical Report CRC-053, SRI Cambridge, 1995. Available at http://www.cl.cam.ac.uk/ jrh13/papers/reflect.ps.gz 
14. J. Harrison. HOL Light: An overview. In S. Berghofer, T. Nipkow, C. Urban, and M. Wenzel, editors, Theorem Proving in Higher Order Logics, volume 5674 of Lecture Notes in Computer Science, pages 60-66. Springer, 2009.

15. L. Henkin. Completeness in the theory of types. Journal of Symbolic Logic, 15:8191, 1950.

16. U. Norell. Towards a Practical Programming Language based on Dependent Type Theory. PhD thesis, Chalmers University of Technology, 2007.

17. U. Norell. Dependently typed programming in Agda. In A. Kennedy and A. Ahmed, editors, TLDI, pages 1-2. ACM, 2009.

18. Plataformatec. Elixir. http://elixir-lang.org/, 2015.

19. W. V. O. Quine. Mathematical Logic: Revised Edition. Harvard University Press, 2003.

20. Rice University Programming Languages Team. Metaocaml: A compiled, type-safe, multi-stage programming language. http://www.metaocaml.org/, 2011.

21. T. Sheard and S. P. Jones. Template meta-programming for Haskell. ACM SIGPLAN Notices, 37:60-75, 2002.

22. A. Stump. Directly reflective meta-programming. Higher-Order and Symbolic Computation, 22:115-144, 2009.

23. W. Taha and T. Sheard. MetaML and multi-stage programming with explicit annotations. Theoretical Computer Science, 248:211-242, 2000.

24. A. Tarski. The concept of truth in formalized languages. In J. Corcoran, editor, Logic, Semantics, Meta-Mathematics, pages 152-278. Hackett, second edition, 1983.

25. The F\# Software Foundation. F\#. http://fsharp.org/ 2015.

26. P. Van Der Walt and W. Swierstra. Engineering proof by reflection in Agda. In R. Hinze, editor, Implementation and Application of Functional Languages, volume 8241 of Lecture Notes in Computer Science, pages 157-173. Springer, 2012. 